\title{
Le projet français PREUVE, projet d'études et de développement pour la lithographie extrême ultraviolet
}

\author{
P. Boher, V. Paret, J.-Y. Robic ${ }^{1}$, R. Marmoret ${ }^{2}$, M. Schmidt ${ }^{3}$, C. Cachoncinlle ${ }^{4}$, \\ R. Geyl $\left.\right|^{5}$, J.-J. Fermé ${ }^{6}$, B. Vidal ${ }^{7}$ et J.-M. Barbiche ${ }^{8}$
}

SOPRA, 26 rue Pierre Joigneaux, 92270 Bois-Colombes, France

${ }^{1}$ CEA/LETI, 17 rue des Martyrs, 38054 Grenoble cedex 9, France

${ }^{2}$ CEA/DAM en lle-de-France, DIF/Département CRE, BP. 12, 91380 Bruyères-le-Châtel, France

${ }^{3}$ CEA-DSM/DRECAM/SPAM, CE-Saclay, bâtiment 522, 91191 Gif-sur-Yvette, France

${ }^{4}$ GREMI-ESPEO, 14 rue d'Issoudun, BP. 6744, 45067 Orléans, France

${ }^{5}$ SAGEM SA, REOSC Products, Division Défense et Sécurité, 61 rue Salvador Allende, 92751 Nanterre, France

${ }^{6}$ SESO, 305 rue Louis Armand, BP. 55000, 13792 Aix-en-Provence, France

${ }^{7}$ LORXN/L3M/LOE, Case 22, Faculté des Sciences de Saint-Jérôme, avenue E. Niemen, 13397 Marseille, France

${ }^{8}$ THALES Laser SA, Domaine de Corbeville, RD. 128, BP. 46, 91401 Orsay cedex, France

\begin{abstract}
Résumé
PREUVE est un projet fédérateur d'acteurs Français (groupes industriels, PME, Laboratoires de Recherche publics et universitaires). Il se situe dans le cadre du développement de la prochaine génération de lithographie pour la micro-électronique avancée, et en particulier la réalisation de circuits aux dimensions sub-micrométriques. Les développements réalisés dans le cadre de PREUVE portent sur plusieurs points clés de la lithographie extrême ultraviolet (EUV)

- Les sources EUV à $13 \mathrm{~nm}$ pour les besoins en lithographie et en métrologie

- Les optiques en réflexion et leurs revêtements multicouches pour les caméras dillumination et de projection

- Les masques (substrats, revêtements multicouches, réticules)

- La métrologie pour les mesures de réflectivité et la détection des défauts des masques.

- Le montage d'un Banc d'Essai de Lithographic (BEL) pour le développement des procédés résines

L'article ci-après résume quelques points clés de ce projet réunis autour d'un descriptif du banc d'essai de lithographie qui fédère l'essentiel des efforts du programme.
\end{abstract}

\section{INTRODUCTION}

\subsection{Les diverses générations de lithographie}

Depuis les débuts de la microélectronique, la puissance des circuits intégrés (IC) n'a cessé de croître principalement grâce à l'augmentation de la capacité d'intégration des circuits. La fameuse loi de Moore selon laquelle la puissance des circuits intégrés double tout les $18 \mathrm{mois}$, n'a pas été remise en défaut depuis 30 ans et semble devoir encore être d'actualité au moins pour les dix prochaines années. Pour cela, un des moteurs principaux est l'amélioration des techniques de définition des circuits ou lithographie, d'abord basée sur la lumière visible el dont les longueurs d'ondes d'utilisation n'ont cessé de décroître ces dernières années pour passer de l'ultraviolet à $248 \mathrm{~nm}$ à lultraviolet profond à $193 \mathrm{~nm}$ et bientôt à $157 \mathrm{~nm}$. Cette demière longueur d'onde parâtt une barrière difficile à franchir car d'une part l'air devient absorbant (l'utilisation de cette longueur d'onde nécessite l'emploi d'un atmosphère exempt d'oxygène et de vapeur d'eau), et d'autre part, les matériaux transparents disponibles pour réaliser les optiques sont peu nombreux et les optiques correspondantes prohibitives. En fait, la prochaine génération de lithographie « classique » à 157mm passera en production en 2003-2004 pour réaliser des circuits de dimensions critiques de l'ordre de $70 \mathrm{~nm}$ selon la SI roadmap [1]. La génération suivante verra elle l'émergence d'un nouveau type de lithographie utilisant des rayons $\mathrm{x}$ « mous s ou EUV (Extrêne UV), pour la réalisation de circuits de taille 
critique inférieure à $70 \mathrm{~nm}$. Cette génération est d'hors et déjà prévue pour 2005 et les grands acteurs de la microélectronique poussent à sont utilisation dés que possible [2]

\subsection{Les optiques réflectives dans l'EUV}

La lithographie EUV est basée sur l'emploi d'optiques réflectives qui sont des cristaux de Bragg artificiels composés d'une alternance périodique de couches dont le contraste d'indice est suffisant pour produire des coefficients réflecteurs importants par interférences constructives pour des angles proches de la normale [3]. Dés le début des années 1980 , avec le développement des techniques de dépôt de couches ultra minces basées sur la pulvérisation ou l'évaporation, la faisabilité de ce type de miroir a été démontré et les couples de matériaux les plus efficaces pour certaines gammes spectrales ont été rapidement mis en évidence, en particulier aux alentours de $13 \mathrm{~nm}$ ou des taux de réflexion supérieurs à $65 \%$ pour le système $\mathrm{Mo} / \mathrm{Si}$ ont été mis en évidence dés 1985 [4]. Ce couple de matériaux est à la base de la technologie sur laquelle s'appuie la lithographie EUV.

\subsection{Les chiffres clés et les principales difficultés}

La résolution (Res) et la profondeur de champ (PDC) d'un système d'imagerie en lithographie sont caractérisées par les deux relations suivantes:

$$
\operatorname{Re} s=k \lambda / O N \quad \text { et } \quad P D C= \pm \lambda /(O N)^{2}
$$

$\lambda$ est la longueur $\mathrm{d}$ 'onde des photons utilisés et ON l'ouverture numérique du système optique. $\mathrm{k}_{1}$ est une constante qui dépend du système optique mais aussi de la résine et du procédé utilisé. Les quelques chiffres du tableau I montrent que les améliorations de résolution se sont faites non seulement en réduisant la longueur d'onde de travail mais aussi en augmentant l'ouverture numérique et donc le coût des optiques ainsi que le procédé. La profondeur de champ n'a aussi pas cessé de baisser et du même coup les contraintes en particulier mécaniques associées n'ont cessés de s'accroître. La réduction d'un ordre de grandeur de la longueur d'onde pour la passage à la génération EUV autorise l'emploi d'optiques d'ouverture plus modeste et des contraintes de procédés plus réduites tout en conduisant à des profondeurs de champ comparables aux générations précédentes. La lithographie EUV n'en constitue pas moins une rupture technologique vis à vis des générations précédentes [5-8], et pas seulement parce que l'on passe d'optiques réfractives à des optiques réflectives mais surtout à cause des spécificités de ces optiques multicouches et la longueur d'onde.

\begin{tabular}{|c|c|c|c|c|c|}
\hline $\begin{array}{c}\text { Type de } \\
\text { lithographic }\end{array}$ & $\begin{array}{c}\text { Longueur } \\
\text { d'onde } \lambda \\
\text { (nm) }\end{array}$ & $\begin{array}{c}\text { Ouverture } \\
\text { numérique ON }\end{array}$ & $k_{1}$ & $\begin{array}{c}\text { Résolution } \\
\text { RES } \\
\text { (nm) }\end{array}$ & $\begin{array}{c}\text { Profondeur de } \\
\text { champ PDC } \\
\text { (nm) }\end{array}$ \\
\hline g-line & 436 & 0.5 & 0.8 & 700 & 1700 \\
\hline -line & 365 & 0.63 & 0.6 & 350 & 920 \\
\hline KrF & 248 & 0.7 & 0.7 & 250 & 690 \\
\hline ArE & 193 & 0.75 & 0.6 & 154 & 343 \\
\hline F2 & 157 & 0.8 & 0.5 & 98 & 245 \\
\hline WUV & $\mathbf{1 3 . 5}$ & 0.2 & 0.8 & $\mathbf{5 0}$ & $\mathbf{3 3 0}$ \\
\hline
\end{tabular}

Tablear 1 : Caracteristiques des différentes générations de lithographies

Un stepper inclura une source EUV avec son système de collection et de focalisation sur le masque, un masque en réflexion basé lui aussi sur les mèmes miroirs, une optique d'imagerie avec un facteur de réduction de l'ordre de 4 pour projeter l'image du masque sur les substrats de silicium. Les difficultés se situent à tous les niveaux. La source EUV doit fournir une puissance EUV atile de l'ordre de $80 \mathrm{~W}$ dans la bande passante des multicouches autour de $13.5 \mathrm{~nm}$. Ceci est nécessaire pour assurer une cadence d'insolation suffisante ( 90 wafers/heure) et à cause de la faible efricacité globale du systeme optique 
( $<3 \%$ pour 9 miroirs à $\sim 70 \%$ ). Elle doit être exempte de débris et non génératrice de pollution à cause de l'extrême sensibilité des revêtements multicouches et du masque. Le masque en réflexion ne doit pas présenter le moindre défaut de réflectance et ceci à une échelle de l'ordre de grandeur de la résolution attendue. Le système optique de réduction est basé sur des optiques aspheriques recouvertes de miroirs multicouches à période graduelle et doit garantir des facteurs de forme de l'ordre de $\lambda / 20$ (soit de l'ordre de deux mono couches atomiques !). Enfin l'ensemble du système doit être maintenu sous ultravide, sans particule et sans contamination.

\subsection{Les principaux thèmes du programme}

Ils sont reportés sur la Figure 1. La réalisation du Banc d'Essai de lithographie (BEL) sert d'élément fédérateur. Afin de réaliser ce démonstrateur qui doit permettre de tester des prototypes de masques et d'effectuer des études de procédés résines, les principaux éléments constitutifs d'un stepper sont étudiés par différents partenaires. Trois types de sources sont étudiés, une source à jet d'agrégats de xénon excité par laser par le CEADRECAM et THALES LASER, une source à décharge capillaire par le GREMI et une source à émission face arrière d'une cible mince solide par le CEADAM. Ces trois sources font l'objet de comptes rendus séparés dans la même conférence et ne seront pas détaillées ici. Chaque source pourra être montée sur le BEL pour effectuer des tests d'insolation. Le système optique complet du BEL a été étudié et réalisé par SAGEM, et les revêtements multicouches associés par l'Université de Marseille comme nous allons le voir ci-après. La maîtrise d'œuvre du BEL est le fait du CEADAM même si le prototype sera installé au LETl. Les masques sont étudiés sous tous les aspects (substrat (SESO), revêtements multicouches (LETI) et réticule (LETI). Enfin des moyens spécifiques de métrologie ont été spécialement développés pour mesurer le taux des réflexions des masques et des optiques (SOPRA) et les défauts des masques (LETI). Quelques uns de ces aspects sont développés ci-après.

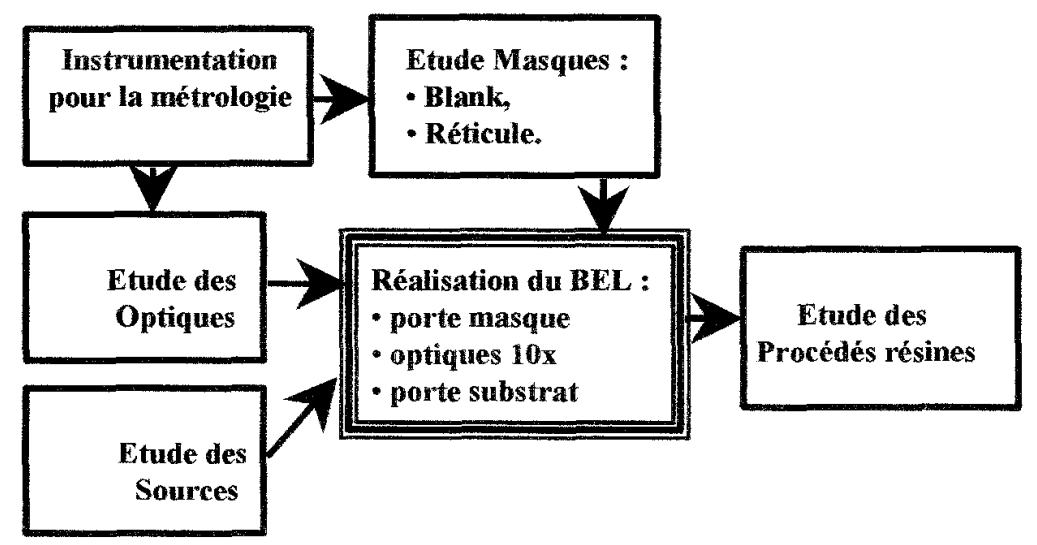

Figure 1: Les principaux thèmes du programme PREUVE

\section{LE BANC D'ESSAI DE LITHOGRAPHIE}

\subsection{Montage optique du BEL}

Dans le projet SAGEM/REOSC réalise l'étude et la fabrication des optiques d'illumination et de projection du banc d'essai de lithographie (BEL). Ceci inclus le développenent et la mise en cuvre de moyens de polissage et de métrologie adaptés ainsi que la maitrise des moyens de montage et d'alignement. Le schéma optique du système est́ représenté sur la figure 2. L'optique de projection et l'optique d'illumination sont des Scharzschild de grande ouverture numérique $(0.32)$ et de grossissement $\times 10$. La taille des miroirs est relativement conséquente (220mm de diametre pour le miroir M8). Le montage symétrique permet d'imager la source sur le wafer, ce qui donnera une taille de zone illuminée de l'ordre de $300 \mu m$ suffisante pour effectuer les tests de résines. La taille des optiques et la précision nécessaire sont 
tout à fait comparables à celles que l'on pourra trouver dans les steppers. Le montage et le réglage des optiques doivent être pris en compte dés la fabrication des miroirs. Les deux principales sources d'erreurs du montage sont justement le montage et la calibration des instruments de contrôle. L'erreur globale est évaluée à un peu plus de $1 \mathrm{~nm}$ rms pour l'optique d'imagerie. Les optiques sont faiblement asphérisées et le montage Scharzschild relativement peu sensible aux erreurs de centrage. Sur un champ de $200 \mu \mathrm{m}$ une précision d'autofocus de $100 \mathrm{~nm}$ est suffisante pour obtenir une zone d'optimum de mise au point d'au moins $10 \times 10 \mu \mathrm{m}$ comme le montre la figure 3 .

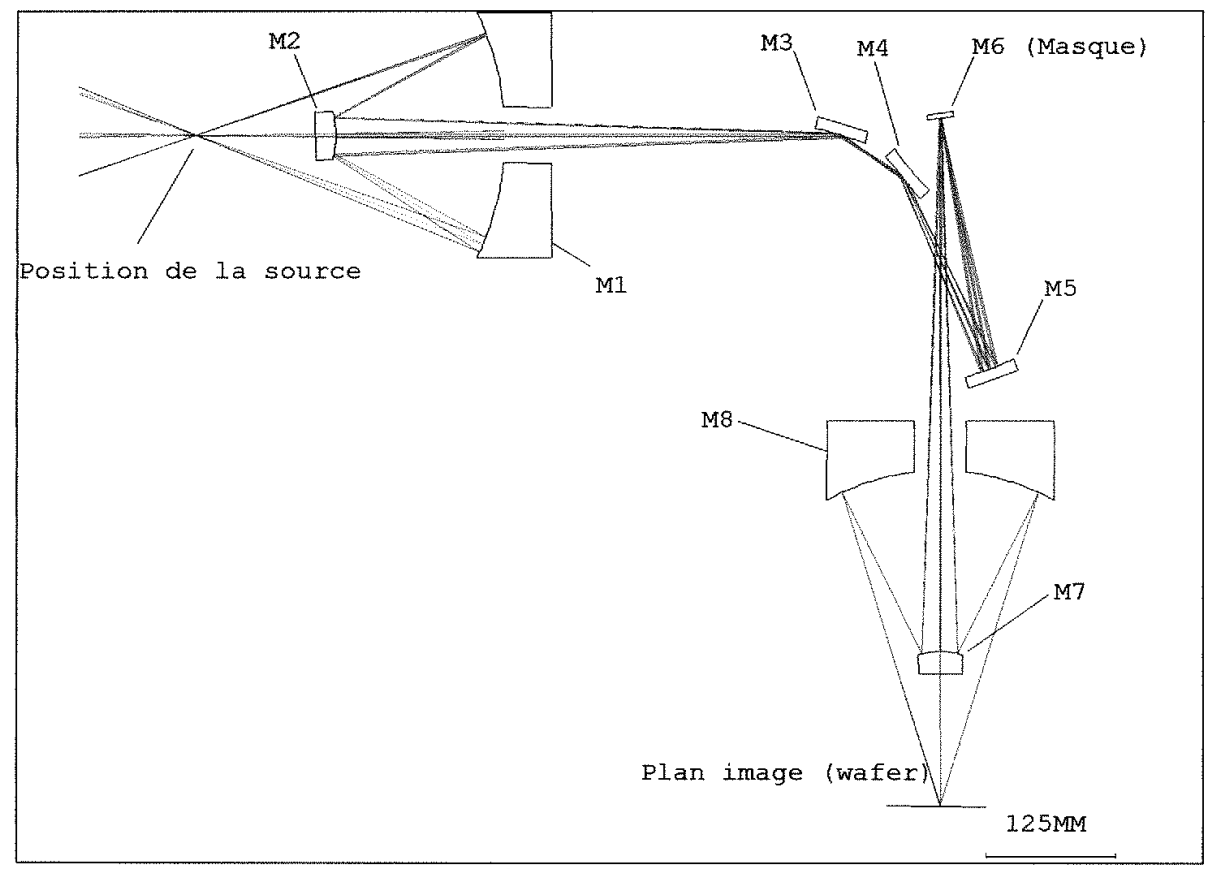

Figure 2 : Schéma du montage optique du BEL

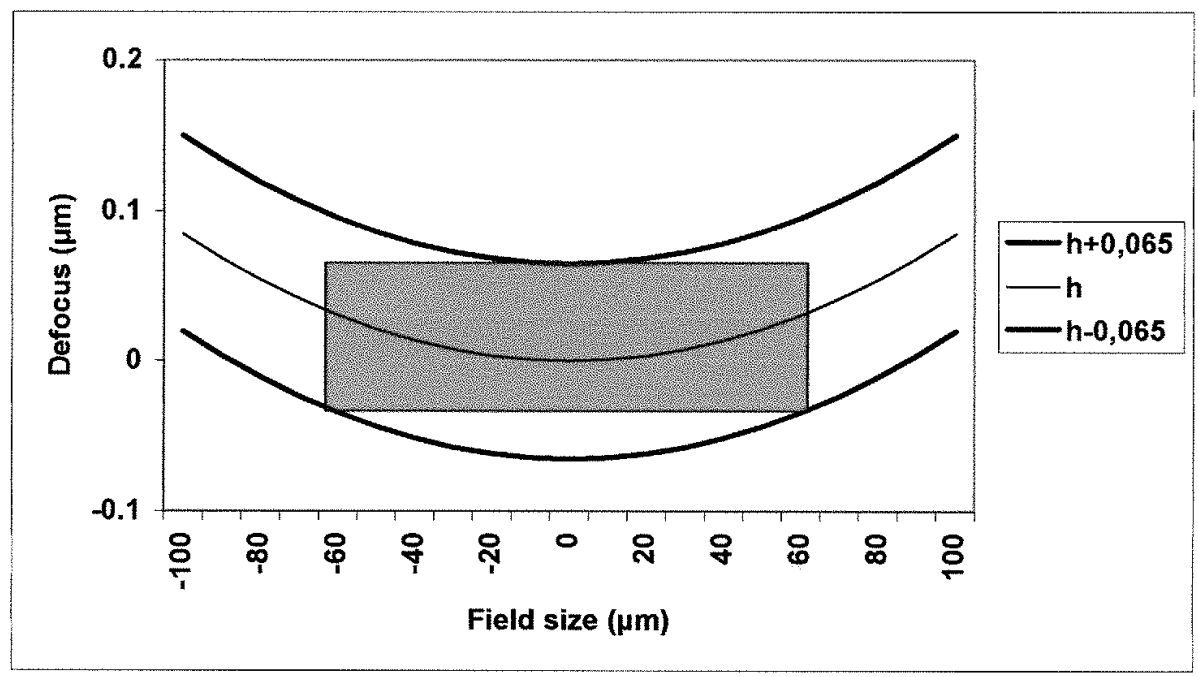

Figure 3 : Fenêtre de travail utile de loptique d'imagerie 


\subsection{Traitement multicouche des optique du BEL}

Un système de pulvérisation radiofréquence spécifique a été développé au LORXN/L2MP/UDESAM dans le cadre du projet pour réaliser les dépôts multicouches à gradient d'épaisseur de toutes les optiques du BEL. Plusieurs études ont déjà été réalisées sur l'optimisation des propriétés des miroirs. Les multicouches sont caractérisées par des mesures de réflectivité EUV (Cf. fig. 4) ainsi que des mesures de réflexion spéculaire et hors spéculaire à $1.54 \mathrm{~A}$. L'analyse des interfaces est complétée par des études de microscope électronique en transmission (TEM). Comme le montre la figure 5 , les couches de molybdène sont partiellement cristallisées alors que les couches de silicium sont totalement amorphes. Des couches de siliciure aux interfaces sont aussi détectées. Des mesures de rugosité de surface sont aussi effectuées à l'aide d'un microscope à force atomique (AFM). Comme on peut le voir sur la figure 4 , des miroirs à incidence quasi normale à $13 \mathrm{~nm}$ ont d'hors et dejà été réalisés avec des performances comparables aux meilleurs résultats publiés dans la littérature (réflectance supérieure à $68 \%$ en incidence quasi-normale[9].

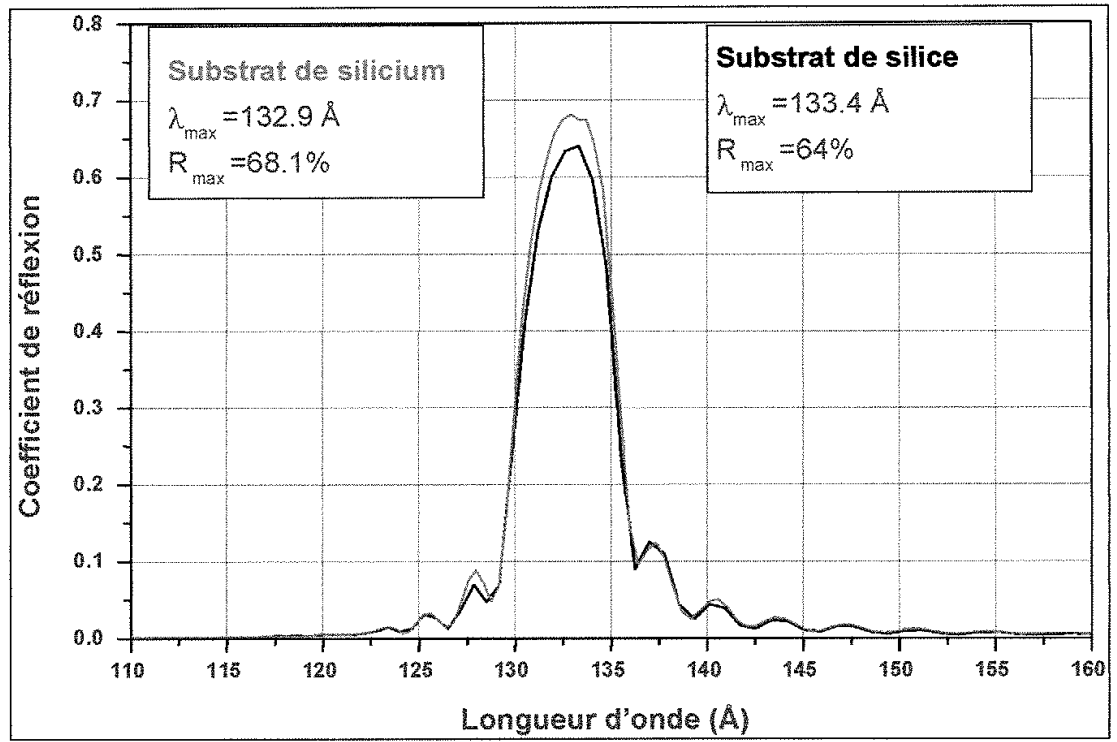

Figure 4 : Taux de réflexion de miroirs Mo/Si déposés sur silicium et silice
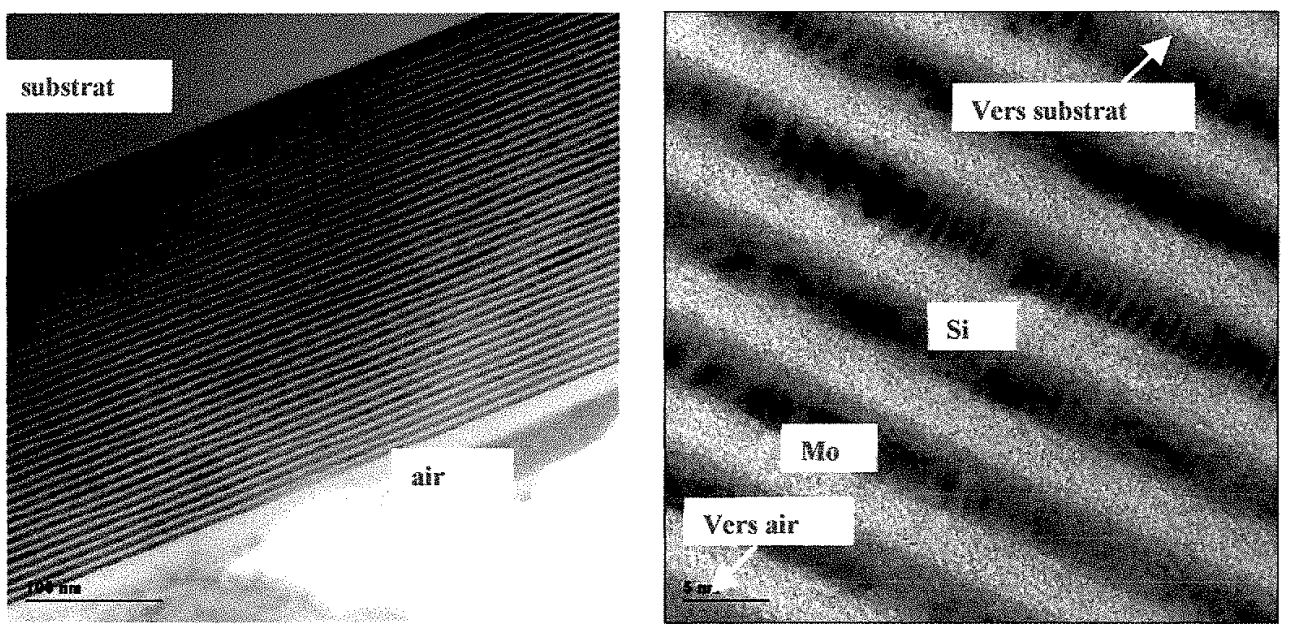

Figure 5 : Microstructure d'une muthicouche MolSi obsenée par microscopie à transmission. 
L'influence de la rugosité du substrat conditionne grandement les performances des miroirs comme le montre la figure 4.

\subsection{Le banc d'essai de lithographie}

Un schéma du système est reporté sur la Figure 6. Le design et le montage du système sont assurés par le CEA/DAM. L'introduction et le transfert des wafers de silicium est réalisé par la partie inférieure d'un microscope à balayage récupéré pour cette occasion. Dans l'enceinte de projection, l'optique d'imagerie est déposée sur un ascenseur constitué de trois mouvements piézoélectriques comme le montre la figure 7. Ceci est nécessaire pour assurer un ajustement précis de l'optique d'imagerie par rapport à la surface du substrat de silicium (cf. figure 3). Un capteur optique de distance permet d'effectuer une pré-mise au point précise. L'enceinte contenant l'optique d'imagerie est isolée mécaniquement de la partie illumination par un soufflet ultravide et une équerre rigide ceci afin d'éviter autant que possible les vibrations mécaniques. Le système est actuellement en cours de montage au CEA/DAM et sera installé au CEA/LETI à la fin du projet.

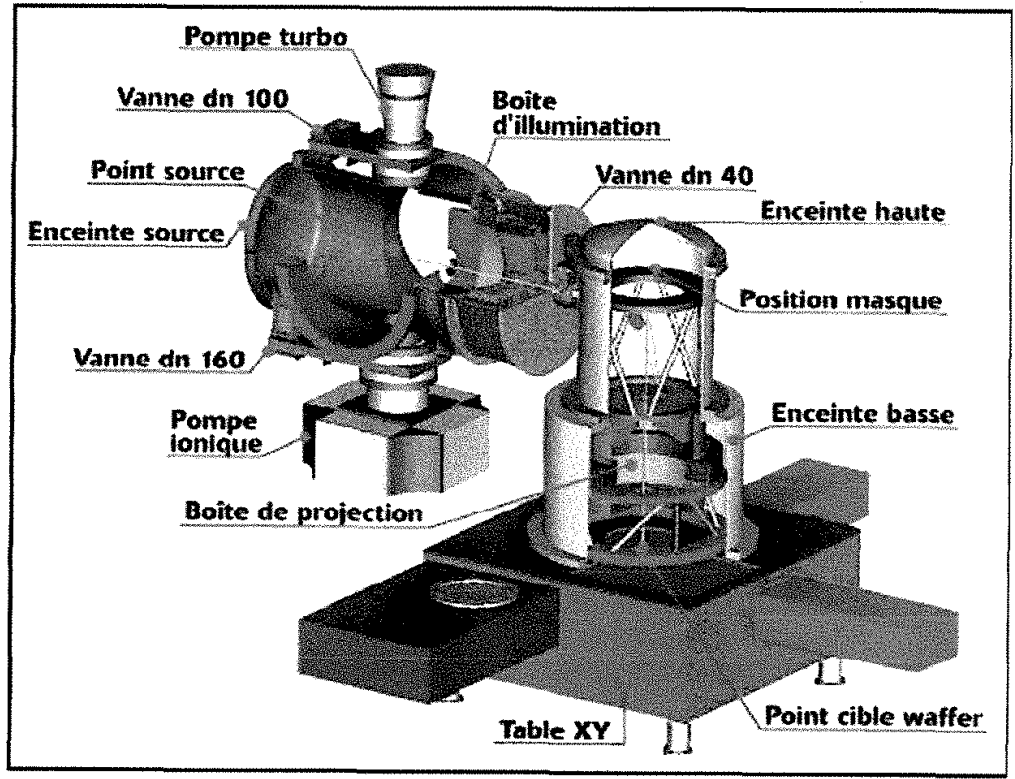

Figure 6 : Schéma de principe du BEL.

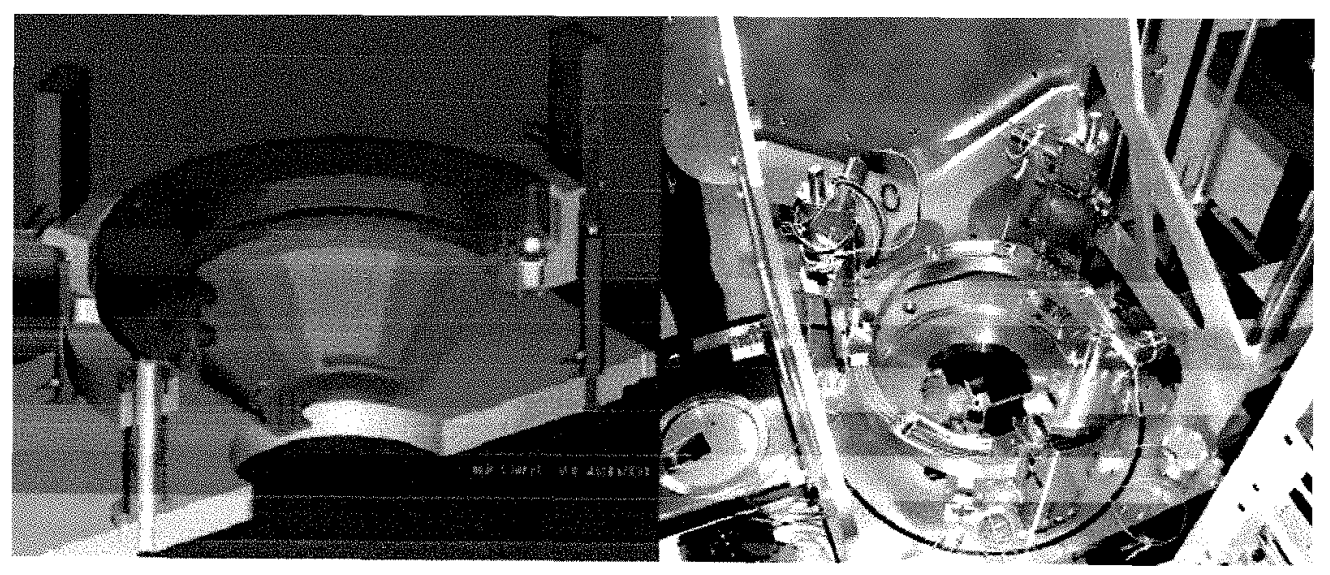

Figure 7 ; Principe et photographie de l'ascenseur de loptique dimagerie 


\section{LA METROLOGIE ET LES TESTS RESINES}

\subsection{Coefficients réflecteurs des masques et des optiques}

SOPRA développe un réflectomètre EUV nécessaire pour mesurer le taux de réflexion des miroirs multicouches des masques et des optiques. Ce type d'instrument sera utilisé de façon routinière pour le contrôle des revêtements des substrats des masques car les procédés de dépôts de multicouches ont besoin d'être contrôlés en permanence pour assurer un accord parfait de la périodicité des empilements avec la longueur d'onde d'urilisation. L'instrument de recherche développement réalisé dans le cadre du projet permet de mesurer les taux de réflexion des miroirs à différents angles d'incidence (de 5 à $90^{\circ}$ ) et dans la gamme spectrale $12-14 \mathrm{~nm}$. Comme le montre la figure 8 , la source de type tube à rayons $\mathrm{x}$ ouvert émet à travers un monochromateur à réseau dans la chambre d'analyse. Le porte échantillon permet à la fois les ajustements nécessaires et une cartographie compatible avec les optiques de grande dimension du BEL $(220 \mathrm{~mm})$. L'échantillon et le détecteur tournent autour d'un axe central qui permet d'ajuster l'angle d'incidence. L'ensemble du système est réalisé en technologie ultravide afin d'éviter toute contamination des masques et des optiques.

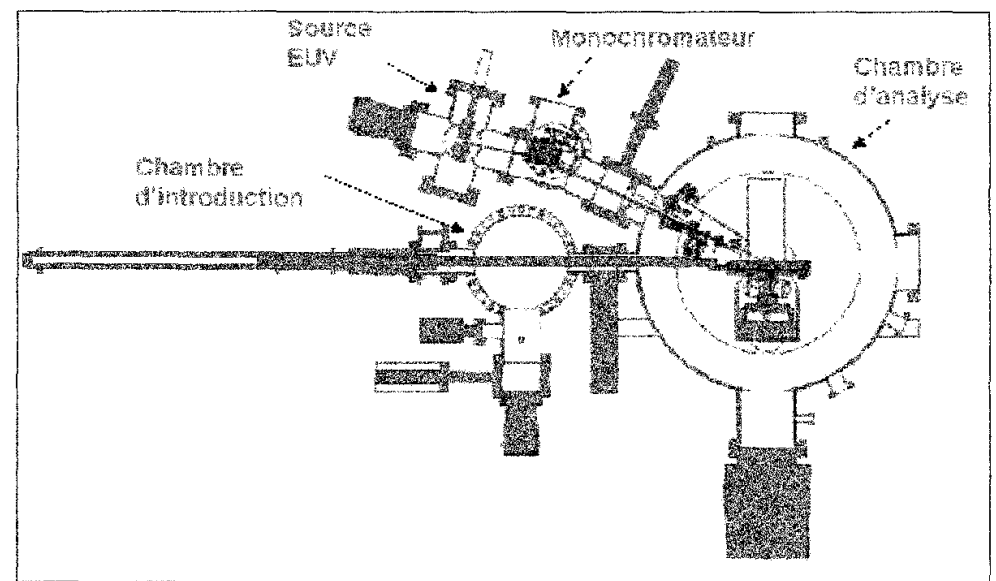

Figure 8 : Schéma du réflectomètre $R \& D$ pour la métrologie des masques et des optiques.

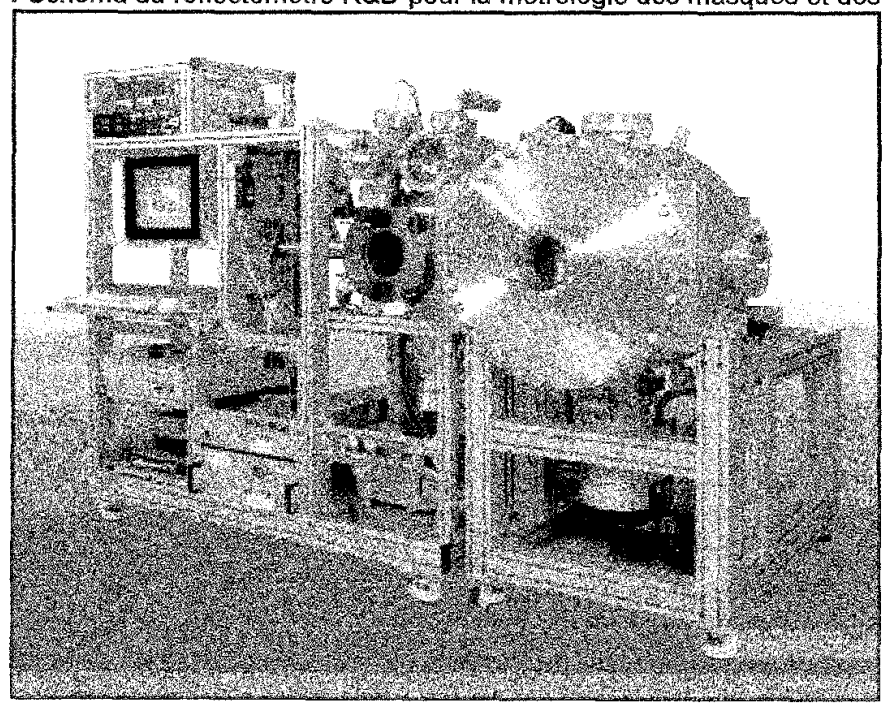

Figure 9 : Photographie du réflectomètre R\&D pour la métrologie des masques et des optiques. 


\subsection{La technologie des masques}

Le principal problème de la technologie des masques est la nécessité de réaliser des objets zéro défaut. En effet, la moindre particule ou défaut de réflectivité sur la surface réflective du masque se traduit par un défaut reproduit sur chaque substrat de silicium. La taille critique de ces défauts est de l'ordre de $100 \mathrm{~nm}$. Ces défauts peuvent avoir plusieurs origines. La présence de particules sur les substrats avant dépôt des multicouches est par exemple généralement amplifiée après dépôt. Le CEA/LETI a breveté puis testé une méthode de fabrication originale qui permet de s'affranchir largement de ce type de défaut (ef. Figure 10). Comme le montre la figure 10, l'idée est d'appliquer la technique bien connu de smart-cut utilisée pour la fabrication du SOI pour aboutir à un masque ou la réflectivité des empilement multicouches concerne les premières et non les dernières couches déposées. Les avantages sont l'emploi de substrat classiques comme le silicium pour le dépôt de l'absorbant (sans contrainte de température) et de la multicouche. Cette technologie commence à être testé par le CEA/LETI et semble prometteuse.

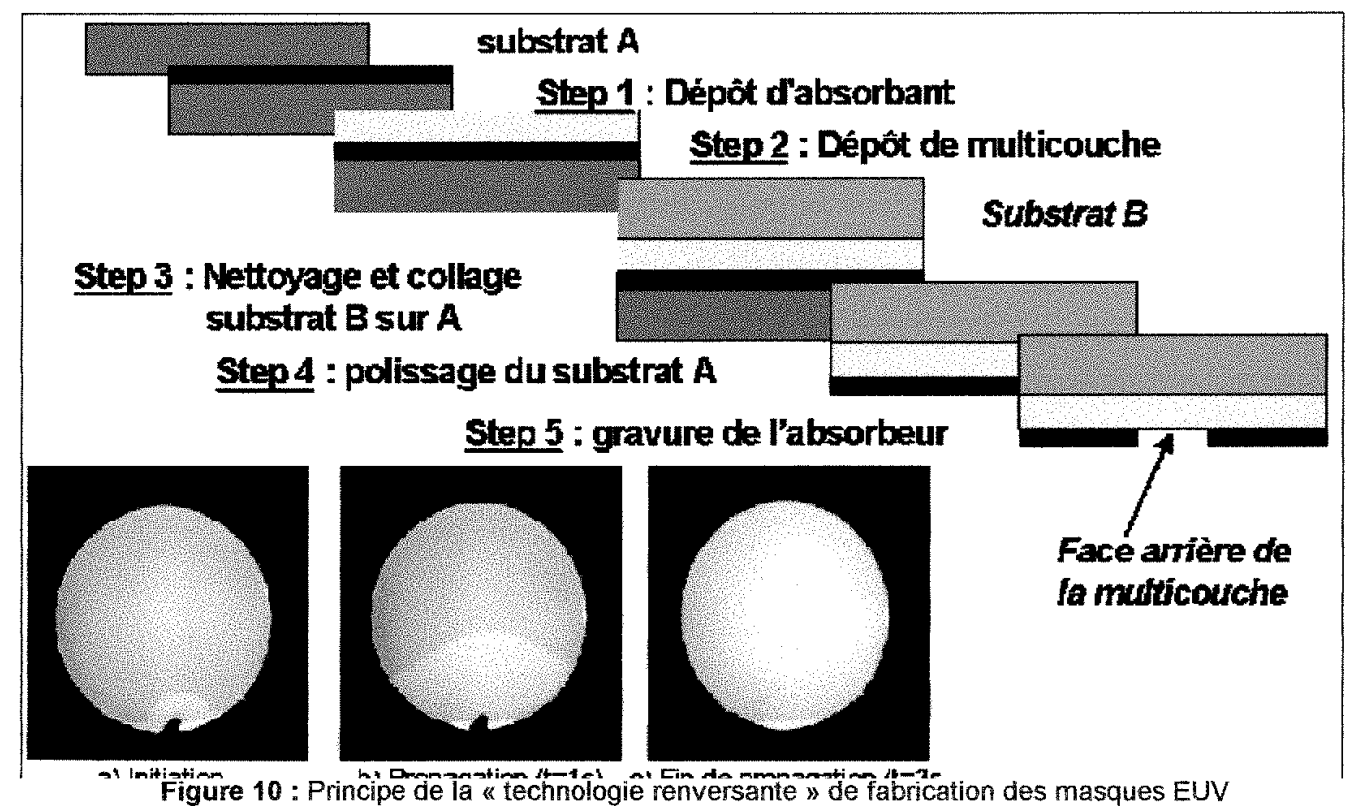

\section{CONCLUSION}

Un des premiers buts de PREUVE qui était de fédérer les competences françaises dans le domaine de la lithographie EUV a déjà été atteint. L'existence du projet au niveau international est reconnue grâce notamment au multiples présentations scientifiques des partenaires dans des conférences internationales. Des premiers résultats significatifs ont déjà été obtenus notamment dans le domaine des sources EUV et des revêtements multicouches. Plusieurs brevets ont aussi été déposés sur les sources et sur l'élaboration des masques. Ce programme doit permettre notamment aux industriels de prendre place sur le marché de la lithographie EUV qui sera un des domaines clés de la microélectronique des prochaines années. THALES LASER, SAGEM/REOSC, SESO et SOPRA ont des atouts à faire valoir sur les sources, les optiques, les substrats des masques et la métrologie. Enfin signalons que ce programme se poursuit dés à présent dans le 
cadre de contrats Européens MEDEA+ avec tous les partenaires majeurs de la microélectronique en Europe.

\section{Remerciements}

Ce travail a été réalisé dans le cadre d'un projet RMNT (Réseau Micro Nano Technologies) soutenu par le ministère de l'industrie.

\section{Références}

[1]. The national technology roadmap for semiconductors - technology needs, 2000 edition, Semiconductor industry association.

[2]. Conférence SPIE, Santa Clara, Février (2002)

[3].E. Spiller, "Soft X-ray optics », SPIE, Belligham, WA (1994)

[4].T.W. Barbee, S. Mrowka, M.C. Hettrick, « Molybdenum-Silicon multilayer mirrors for normal incidence in the Extreme Ultraviolet », Appl. Opt., 24, 883 (1985)

[5].A.M. Hawryluk, L.G. Seppala, J. Vac. Sci. Technol., B6, 2162 (1988)

[6].W.T. Silfvast, O.R. Wood, II, Microelectron. Eng., 8, 3 (1988)

[7].H.Kinoshita, K/ Kurhara, Y.Ishii, Y. Torii, J. Vac. Sci. Technol, B7, 1648 (1989)

[8].C.W. Gwyn, R.H. Stulen, D.W. Sweeney, D.T. Attwood, « Extreme ultraviolet Lithography », J. Vac. Sci, Techn.., B16 (1998)

[9].E. Louis, «E-beam coating technology for EUVL », 2eme annual workshop on EUV lithography, San Francisco, october 17-19 (2000) 